\title{
BMJ Open Effect of acetazolamide on obstructive sleep apnoea in highlanders: protocol for a randomised, placebo-controlled, double-blinded crossover trial
}

\author{
Lu Tan, ${ }^{1}$ Michael Furian, ${ }^{2}$ Taomei Li, ${ }^{1}$ Xiangdong Tang (D) ${ }^{1}$
}

To cite: Tan L, Furian M, Li T, et al. Effect of acetazolamide on obstructive sleep apnoea in highlanders: protocol for a randomised, placebocontrolled, double-blinded crossover trial. BMJ Open 2022;12:e057113. doi:10.1136/ bmjopen-2021-057113

- Prepublication history for this paper is available online. To view these files, please visit the journal online (http://dx.doi. org/10.1136/bmjopen-2021057113).

Received 05 September 2021 Accepted 11 February 2022

Check for updates

(c) Author(s) (or their employer(s)) 2022. Re-use permitted under CC BY-NC. No commercial re-use. See rights and permissions. Published by BMJ.

${ }^{1}$ Sleep Medicine Center, Department of Respiratory and Critical Care Medicine, Translational Neuroscience Center, State Key Laboratory of Biotherapy, West China Hospital, Sichuan University, Chengdu,

China

${ }^{2}$ Sleep Disorders Center, Department of Respiratory Medicine, University Hospital of Zurich, Zurich, Switzerland

Correspondence to

Dr Xiangdong Tang;

2372564613@qq.com

\section{ABSTRACT}

Introduction Obstructive sleep apnoea (OSA) is a highly prevalent disease that causing systemic hypertension. Furthermore, altitude-dependent hypobaric hypoxic condition and Tibetan ethnicity have been associated with systemic hypertension independent of OSA, therefore patients with OSA living at high altitude might be at profound risk to develop systemic hypertension. Acetazolamide has been shown to decrease blood pressure, improve arterial oxygenation and prevent high altitude periodic breathing in healthy volunteers ascending to high altitude and decrease blood pressure in patients with systemic hypertension at low altitude. However, the effect of acetazolamide on 24-hour blood pressure, sleepdisordered disturbance and daytime cognitive performance in patients with OSA permanently living at high altitude has not been studied.

Methods and analysis This study protocol describes a randomised, placebo-controlled, double-blinded crossover trial. Highland residents of both sexes, aged 30-60 years, Tibetan ethnicity, living at an elevation of $3650 \mathrm{~m}$ and apnoea-hypopnoea index over 15/hour will be included. Participants will be randomly assigned to a $2 \times 2$ week treatment period starting with $750 \mathrm{mg} /$ day acetazolamide followed by placebo treatment or vice versa, separated by a 1-week wash-out phase. Clinical assessments, 24-hour ambulatory blood pressure monitoring (ABPM), polysomnography (PSG), near-infrared spectroscopy, nocturnal fluid shift and cognitive performance will be assessed before and at the end of each treatment period. The primary outcome will be the difference in 24-hour mean blood pressure between acetazolamide therapy and placebo; secondary outcomes will be the difference in other 24-hour ABPM-derived parameters, PSG-derived parameters, cognitive performance and overnight change in different segments of fluid volume between acetazolamide therapy and placebo. Accounting for potential dropouts, 40 participants will be recruited.

Ethics and dissemination The protocol was approved by the West China Hospital of Sichuan University Biomedical Research Ethics Committee. Recruitment will start in spring 2022. Dissemination of the results include presentations at conferences and publications in peerreviewed journals.

Trial registration number ChiCTR2100049304.
Strengths and limitations of this study

- This randomised, placebo-controlled, doubleblinded crossover trial will provide robust findings on the effect of acetazolamide on blood pressure in patients with obstructive sleep apnoea (OSA) living at high altitude - a population rarely investigated so far.

- Blood pressure will be assessed by the gold standard of 24-hour ambulatory blood pressure monitoring and will provide valuable insights into day and night blood pressure alterations.

- Underlying mechanisms and consequences related to blood pressure changes will be assessed by nocturnal fluid shift, sleep-disordered breathing, cognitive performance and cerebral homeostasis measurements.

- Findings from the applied 2-week acetazolamide treatment will be novel, but will not provide enough evidence to directly implement long-term acetazolamide treatment for patients with OSA living at high altitude.

- Findings will be obtained in Tibetan highlanders, therefore, antihypertensive effects of acetazolamide might vary in other high altitude populations.

\section{INTRODUCTION}

Obstructive sleep apnoea (OSA) is characterised by repetitive collapse of the upper airway, resulting in intermittent cessation of airflow, hypoxemia and sleep disturbance. ${ }^{1}$ In recent years, the prevalence of OSA increased and has been reported to be $34 \%$ in men and $17 \%$ in women aged $30-70$ years. ${ }^{23}$ The pathophysiology of OSA includes anatomic and nonanatomic factors. ${ }^{4}$ Anatomic factors include shorter and more vertical mandible, tonsillar and adenoid hypertrophy and unusual large tongue, however, overnight fluid shift from the legs to the upper body is receiving increased attention. Accumulated fluid in the veins and soft tissue of the leg from daytime orthostatic gravity may shift to the neck while sleeping in supine position. This fluid 
transfer causes a reduction in pharyngeal size, increased pharyngeal resistance and upper airway collapsibility. ${ }^{5}$ Previous studies found that patients with chronic venous insufficiency, lower limbs lymphedema, renal failure and heart failure associated with fluid overload had higher prevalence of OSA. ${ }^{5}$ Additionally, OSA severity has been correlated with volume of nocturnal fluid transfer. ${ }^{7}$ Aerobic exercise, compression stockings and manipulation of salt or fluid intake may reduce the severity of OSA through the decrease of leg fluid accumulation. ${ }^{5}$

It is well known that OSA is associated with cardiovascular disorders including systemic arterial hypertension. Therefore, it has been reported that OSA is associated with a higher prevalence of non-dipping circadian patterns probably due to apnoea and hypopnoea-related blood pressure swings during sleep and daytime hypertension. ${ }^{8}$ In accordance with these findings, a prevalence of hypertension of $46 \%-53 \%$ has been reported in patients with moderate to severe OSA, ${ }^{9}$ and a pooled mean OR of 1.18, 1.32 and 1.56 has been estimated for developing hypertension for mild, moderate and severe OSA, respectively. ${ }^{10}$

With increasing altitude, the barometric pressure falls and the partial pressure of inspired oxygen decreases proportionally. High altitude is defined as an altitude over $2500 \mathrm{~m}$ with the main characteristic of hypobaric hypoxia. ${ }^{11}$ It is estimated that over 140 million people are permanently living at high altitude and the prevalence of OSA may be similar or even higher under these hypobaric hypoxic conditions due to hypoxia-dependent instability in ventilatory control. ${ }^{12}$ Accordingly, previous studies in long-term acclimatised high altitude residents reported elevated prevalence of sleep-disordered breathing including OSA compared with lowlanders. ${ }^{13} 14$

The higher prevalence of OSA, the sympathetic overactivity induced by hypobaric hypoxia and cold weather at high altitude are cardiovascular risk factors. These and other risk factors contribute to acute and persistent altitude-induced elevations of blood pressure. ${ }^{15-18}$ This has been confirmed by studies in Chinese workers, revealing a prevalence of hypertension and prehypertension of $26.7 \%$ and $41.3 \%$, respectively. ${ }^{19}$ Moreover, systematic review and meta-analysis in highlanders of Tibetan origin estimated a $2 \%$ increase in the prevalence of hypertension per $100 \mathrm{~m}$ increased altitude of living, or a $17 \mathrm{~mm} \mathrm{Hg}$ and $9.5 \mathrm{~mm} \mathrm{Hg}$ increase in average systolic and diastolic blood pressure per $1000 \mathrm{~m}$ increased altitude of living, respectively. ${ }^{21}$ These findings were confirmed and extended by Bilo et al, showing a higher prevalence of nocturnal hypertension in highlanders. ${ }^{22}$

Acetazolamide, a carbonic anhydrase inhibitor with diuretic effects, is the recommended pharmaceutical measure to prevent acute mountain sickness in trekkers ascending to high altitudes. ${ }^{23}{ }^{24}$ Apart from the preventive effect of acetazolamide against acute mountain sickness, this medication has been shown to reduce blood pressure in healthy persons and patients with OSA at low and high altitude. ${ }^{25} 26$ The antihypertensive effect of acetazolamide on blood pressure may be related to changes in sleep-disordered breathing or changes in fluid shift among others. Indeed, studies have confirmed that diuretics are efficient in decreasing blood pressure and apnoea-hypopnoea index (AHI) in obese patients with hypertension and patients with uncontrolled or resistant hypertension. ${ }^{27-29}$ In patients with comorbid OSA and hypertension, acetazolamide effectively reduced blood pressure and the severity of sleep-disordered breathing. ${ }^{30} 31$ When travelling to high altitude, one study reported less altitude-induced systolic and diastolic blood pressure elevations in healthy persons acutely exposure to $4559 \mathrm{~m}$ under acetazolamide compared with placebo. ${ }^{18}$ Moreover, altitude-induced sleep-disordered breathing also improved under acetazolamide. ${ }^{32}$ These observations were confirmed in patients with OSA ascending to moderate altitude, where they benefitted from 500 to $750 \mathrm{mg} /$ day acetazolamide due to a decrease in AHI, improved arterial oxygenation and prevention of excessive blood pressure elevations. ${ }^{33} 34$

Based on these studies, acetazolamide seems to be effective in preventing acute altitude-induced blood pressure elevations and sleep-disordered breathing in healthy persons and in patients with OSA or hypertension. However, the effect of acetazolamide on blood pressure and sleep-disordered breathing in highlanders with OSA remains to be elucidated. Therefore, the objective of this study will be to investigate the effect of acetazolamide on 24-hour blood pressure in Tibetan highlanders with OSA. Further objectives will be to evaluate the effect of acetazolamide on sleep-disordered breathing and daytime cognitive performance. Our primary hypothesis is that acetazolamide therapy reduces the 24-hour mean blood pressure in Tibetan highlanders with OSA compared with placebo. Secondary hypotheses are that acetazolamide reduces the AHI, improves oxygen saturation and daytime cognitive performance compared with placebo.

\section{METHODS AND ANALYSIS}

\section{Study design and setting}

This study will be a randomised, double-blinded, placebo-controlled cross-over trial evaluating the effect of acetazolamide on 24-hour blood pressure, nocturnal breathing pattern and daytime cognitive performance in highlanders with moderate to severe OSA living in the Lhasa area $(3650 \mathrm{~m}$, barometric pressure of $649 \mathrm{mbar})$. Participants will receive acetazolamide or placebo for 2 weeks each, in a random order and separated by a 1-week wash-out period in-between. Assessments of the primary and secondary outcomes will be performed before and at the end of each 2-week treatment period with acetazolamide or placebo, respectively (figure 1). Examinations will be carried out in the Lhasa People's Hospital, Lhasa, Tibet Autonomous Region, China, from spring until autumn 2022. 


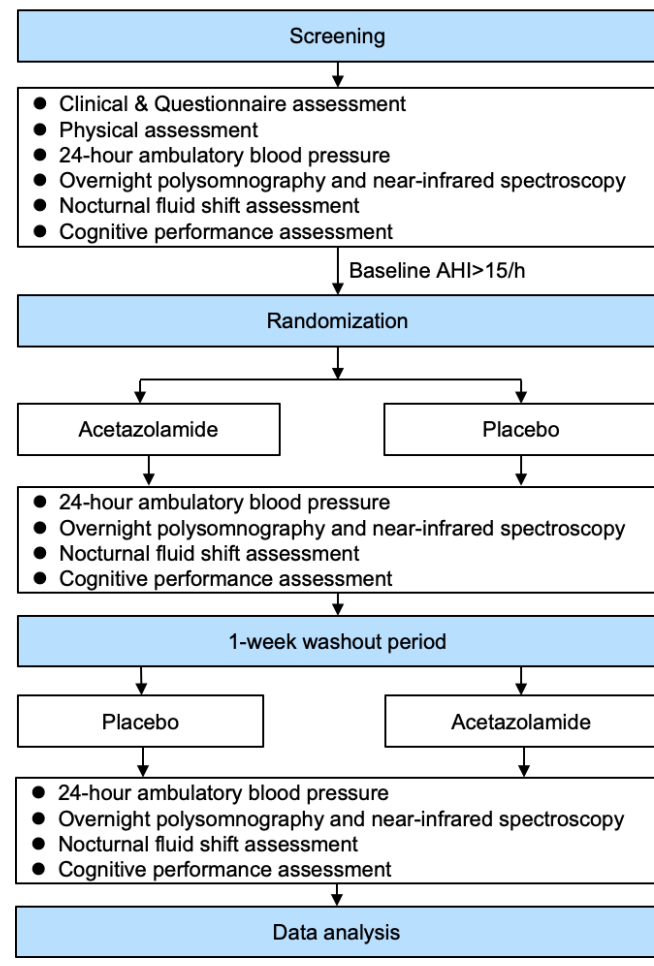

Figure 1 Time schedule of screening, interventions, assessments and visits of participants. AHI, apnoeahypopnoea index.

\section{Participants}

Word of mouth propaganda, social media and electronic advertisement will be used to recruit the required number of untreated highlanders with OSA from the general population. Residents of Tibetan ethnicity, both sexes, aged 30-60 years living in Lhasa for over 10 years who reported snoring or witnessed apnoea during sleep or daytime sleepiness will be invited for a screening visit. Persons presenting an AHI over 15 events/hour according to a baseline polysomnography (PSG) will be included and randomised. Exclusion criteria will be those with diagnosed hypertension who are currently taking antihypertensive medication or undiagnosed moderate to severe hypertension (systolic blood pressure $>160 \mathrm{~mm}$ $\mathrm{Hg}$ and diastolic blood pressure $>100 \mathrm{~mm} \mathrm{Hg}$ ); any known cardiovascular diseases such as angina, coronary heart disease or atrial fibrillation, uncontrolled diabetes (fasting blood glucose $>6.5 \mathrm{mmol} / \mathrm{L}$ ) or previous stroke; any known chronic respiratory disease and/or chronic hypercapnia respiratory failure; liver dysfunction or renal dysfunction; any known sleep disorders, such as insomnia, narcolepsy, restless leg syndrome or rapid eye movement behaviour disorder; chronic mountain sickness (Qinghai chronic mountain sickness (CMS) score over 6 points including elevated haemoglobin concentration) or high-altitude pulmonary hypertension; current continuous positive airway pressure therapy or mandible advancement therapy; history of surgery such as uvulopalatopharyngoplasty to relieve OSA; pregnancy or lactation.
Participants who were exposed to altitudes $<1500 \mathrm{~m}$ or $>5000 \mathrm{~m}$ in the previous 6 months for more than 1 week will not be eligible for the study. Furthermore, participants who are taking concomitant medication that may interfere with acetazolamide (ie, high dose of acetylsalicylic acid or additional diuretics among others) will also be excluded.

\section{Randomisation and intervention}

Participants with an initial AHI over 15 events/hour assessed by baseline PSG will be randomly assigned to 2 weeks acetazolamide followed by 2 weeks placebo treatment, or vice versa, with a 1:1 allocation ratio as per computer-generated schedule (MinimPy) minimising for gender and AHI severity ( $<30$ events/hour and $\geq 30$ events/hour). The applied dosage of acetazolamide will be $750 \mathrm{mg} /$ day $(250 \mathrm{mg}$ in the morning and $2 \times 250 \mathrm{mg}$ in the evening) or identically looking placebo capsules. A wash-out period of 1 week in-between will avoid any carryover effects between treatments. During the complete study, participants will keep track and report any drugrelated side effects or adverse events.

Study protocol adherence will be controlled by individual telephone visits on the first 3 days of each treatment period and face-to-face visits after 1 week of treatment. During these visits, the number of remaining capsules, the importance of the study, individual needs and questions will be discussed. Participants who are not willing to continue with the study, who choose other treatment options (continuous positive airway pressure or mandibular advancement therapy) or who could not tolerate acetazolamide-related side effects will be withdrawn from the study after a final medical evaluation by the chief investigators. Participants who have serious adverse events (metabolic acidosis, hypokalaemia, renal colic, haematuria and exfoliative dermatitis) will immediately terminate treatment and will receive the corresponding therapies. Participants with serious adverse events will have follow-up screening every week in the first month and every 2 weeks in the following 2 months.

\section{Blinding}

An independent pharmacist will prepare identically looking active and placebo capsules labelled with 4-digit codes. The list of codes will be kept confidential to investigators and participants until data acquisition and analysis has been completed. The person in charge for the statistical analysis will not be involved in data acquisition and will not be aware of the treatment allocation. Un-blinding of the treatment will only be allowed when a participant experiences serious adverse events, then an independent investigator will reveal the current treatment of this participant.

\section{Assessments}

The assessments at baseline, after the 2-week treatment periods are summarised in table 1 . 
Table 1 Summary of the assessments

\begin{tabular}{|c|c|c|c|}
\hline \multirow[b]{2}{*}{ Assessments } & \multirow[b]{2}{*}{ Baseline } & \multicolumn{2}{|l|}{ Randomisation } \\
\hline & & First 2-week period & Second 2-week period \\
\hline Informed consent & $\sqrt{ }$ & & \\
\hline Clinical and questionnaire assessment & $\sqrt{ }$ & & \\
\hline Physical assessment & $\sqrt{ }$ & $\sqrt{ }$ & $\sqrt{ }$ \\
\hline 24-hour ambulatory blood pressure monitoring & $\sqrt{ }$ (before PSG) & $\sqrt{ }$ (before PSG) & $\sqrt{ }$ (before PSG) \\
\hline Overnight polysomnography (PSG) & $\sqrt{ }$ & $\sqrt{ }$ & $\sqrt{ }$ \\
\hline Near-infrared spectroscopy & $\sqrt{ }$ (with PSG) & $\sqrt{ }$ (with PSG) & $\sqrt{ }$ (with PSG) \\
\hline Nocturnal fluid shift assessment & $\begin{array}{l}\sqrt{ } \text { (before and after } \\
\text { PSG) }\end{array}$ & $\sqrt{ }$ (before and after PSG) & $\sqrt{ }$ (before and after PSG) \\
\hline Cognitive performance assessment & $\sqrt{ }($ after PSG) & $\sqrt{ }($ after PSG) & $\sqrt{ }($ after PSG) \\
\hline
\end{tabular}

Sleep diary and adverse events report will be filled every day after randomisation. Physical assessment includes blood routine test, liver or renal function test, spirometry and arterial blood gas analysis.

\section{Clinical assessment and questionnaire}

A detailed medical history and clinical evaluation will be obtained including information about the ethnicity, altitude of residence, previous and current illnesses and medications, and smoking and alcohol habits. Height, weight, vital signs, resting blood pressure and pulse oximetry $\left(\mathrm{SpO}_{2}\right)$ will also be obtained.

Berlin questionnaire ${ }^{35}$ and the modified Epworth sleepiness scale ${ }^{36}$ will be used to evaluate the risk of having OSA and daytime sleepiness. The Leeds sleep evaluation questionnaire containing 10 items $^{37}$ and a $100 \mathrm{~mm}$ visual analogue scale will be used to evaluate subjective sleep quality in the morning after sleep assessments.

\section{Physical assessment}

A venous blood sample will be drawn to obtain haemoglobin, fasting blood glucose, alanine aminotransferase, aspartate transaminase, albumin and creatinine in order to exclude participants with chronic mountain sickness or uncontrolled diabetes, liver and renal dysfunction. Spirometry will be carried out according to published standards $^{38}$ and parameters such as forced vital capacity and forced expiratory volume in one second will be measured to exclude participants with respiratory diseases. An arterial blood sample will also be drawn in the morning after sleep assessments to obtain $\mathrm{pH}, \mathrm{PaO}_{2}, \mathrm{PaCO}_{2}$ and haemoglobin concentration.

\section{4-hour ambulatory blood pressure monitoring}

The 24-hour ambulatory blood pressure monitoring will be performed by Spacelabs recorders (Spacelabs Healthcare, Hertfordshire, UK), 1 day before PSG. The cuff will be positioned on the non-dominant arm (right in the left-handed participants) to limit the interference of arm movement. The skin border will be marked with ink so that the cuff could be repositioned in case of movement. The cuff will be programmed to inflate every $15 \mathrm{~min}$ between 07:00 and 23:00 (daytime) and every $30 \mathrm{~min}$ between 23:00 and 07:00 (nighttime). The awake-asleep periods will be defined from 07:00 to 22:59 and 23:00 to
06:59, respectively. Therefore, the participants will be instructed to respect these awake-asleep periods during their 24-hour ambulatory blood pressure assessments. The mean, systolic and diastolic arterial blood pressure during 24 hours as well as for daytime and nighttime periods will be calculated.

\section{Sleep studies}

Overnight PSG (Alice 6; Respironics, Murrysville, USA) will be performed according to standard techniques including six electroencephalographic (EEG) leads (F3-A2, F4-A1, C3-A2, C4-A1, O1-A2, O2-A1), two electrooculographic leads (ROC-A1, LOC-A2), four electromyographic leads (two submental and bilateral tibialis anterior), oral thermistor, nasal pressure transducer, thoracic and abdominal inductance plethysmography, finger pulse oximetry, ECG, snoring and body position.

Thirty-second epochs from lights off to lights on will be analysed and sleep stages, arousals and respiratory events will be scored according to American Academy of Sleep Medicine guidelines (V.2.3). ${ }^{39}$ An apnoea will be defined as more than $90 \%$ reduction in airflow for at least $10 \mathrm{~s}$. Obstructive apnoea or central apnoea will be scored based on whether or not it is associated with continued or increased inspiratory effort throughout the entire period of absent airflow. Mixed apnoea will be scored as obstructive apnoea to be consistent with a previous published study. ${ }^{40}$ Hypopnoea will be defined as $30 \%$ or more reduction for at least $10 \mathrm{~s}$ associated with at least $3 \%$ reduction in oxygen saturation or an arousal. Obstructive hypopnoea will be scored if there is snoring during the event or there is increased inspiratory flattening of the nasal pressure compared with baseline breathing or there is an associated thoraco-abdominal paradox that occurs during the event but not during pre-event breathing. Central hypopnoea will be scored if the hypopnoea does not meet any criteria for obstructive hypopnoea. Since acetazolamide could increase oxygen saturation, which might influence the magnitude of $\mathrm{SpO}_{2}$ dips due to the sigmoidal shape 
of the oxygen dissociation curve, exploratory analysis will additionally score hypopnoeas according to 1999 AASM Chicago Consensus Report that requires a $\geq 50 \%$ amplitude reduction of airflow for at least $10 \mathrm{~s}$ but does not include an oxygen desaturation criterion.

\section{Near-infrared spectroscopy}

Near-infrared spectroscopy (NIRS) will be used to measure cerebral tissue oxygenation. NIRS will be performed by a 4-channel device (ENGINMED, EGOS600A, Jiangsu, China) emitting at wavelengths of 760, 810 and $840 \mathrm{~nm}$. Pairs of emission and detection optodes will be placed bilaterally, high on the forehead, where sinuses are avoided, at the midpoint between Fp1-F3 landmarks of the international EEG 10-20 system electrode placement. Optodes will be secured with double adhesive tape and a self-adhesive elastic dressing. Placements will be identical between assessments. Cerebral tissue oxygenation and the relative changes from awake state before lights off to different sleep stages of oxygenated, deoxygenated and total haemoglobin concentration, and tissue haemoglobin index will be assessed. NIRS measurements will be performed using a sampling rate of $1 \mathrm{~Hz}$ during the whole night together with overnight PSG.

\section{Nocturnal fluid shift assessment}

Bioelectrical impedance is a well validated and highly reproducible (accuracy within $0.5 \%$, repeatability within $0.3 \%$, test-retest correlation $>95 \%$ ), non-invasive technique to estimate fluid volume of tissues. ${ }^{41}$ Bioelectric impedance has been used to measure leg fluid volume in response to various interventions. ${ }^{42-44}$ In the current study, nocturnal fluid shift assessments will be performed using the InBody S10 system (InBody, Seoul, South Korean). Participants will be asked to empty their bladders and rest in bed for at least $30 \mathrm{~min}$ in the evening of the PSG assessments. The second measurement will be carried out within $15 \mathrm{~min}$ of awakening, before eating or drinking. During these measurements, we will assess weight, body mass index, neck circumference, left and right arm fluid volumes, left and right leg fluid volumes and trunk fluid volumes. Fluid shift volume $(\Delta, \mathrm{mL})$ of each segment will be calculated by pre-sleep fluid volume minus post-sleep fluid volume. The fluid volume of the head and neck will be calculated with the formula: $\Delta$ left leg $+\Delta$ right leg $-\Delta$ left arm $-\Delta$ right arm $-\Delta$ trunk according to a previous study. ${ }^{45}$

\section{Cognitive performance assessment}

Psychomotor vigilance test (PVT)

Vigilance will be assessed by psychomotor vigilance testing, which is sensitive for effects of sleep restriction/disturbance and hypoxia. ${ }^{46}$ Before the baseline assessments, participants will receive the opportunity to perform a familiarisation testing consisting of three full tests. Baseline testing will then be carried out in the morning using a self-made computer programme. Participants will be asked to sit in a silent room and will be instructed to tap 'space' on the keyboard as fast as possible after the appearance of a red dot on the screen. The test will last for $10 \mathrm{~min}$ and the interval of the consecutive appearances of the red dot is randomised by the computer. The reaction time of each tap will be recorded and the mean reaction time and $1 /$ reaction time will be calculated.

Trail making tests A and B (These two tests were also part of cognitive performance assessment, so the font size of this title should be the same as "psychomotor vigilance test". As I could not change the font size of the title by myself, so I add the comment here)

During the trial making test A, participants will be instructed to connect the consecutive numbers from 1 to 25 as fast as possible. During the trial making test B, participants will be instructed to connect the numbers and corresponding letters as fast as possible, (1 to A, A to 2, 2 to $\mathrm{B}$ and so on). The total time to complete the tasks will be recorded.

\section{Sleep diary}

Participants will record their sleeping habits in a sleep diary, which includes information about time of drugintake, time of going to bed and time of leaving the bed in the morning. Additionally, time and duration of daytime napping will be recorded in the sleep diary.

\section{Outcomes}

The primary outcome of this study will be the difference in 24-hour mean blood pressure from the 2-week acetazolamide compared with placebo treatment. Secondary outcomes will be the difference in other parameters derived from 24-hour ambulatory blood pressure monitoring (24-hour systolic and diastolic blood pressure, daytime mean, systolic and diastolic blood pressure, nighttime mean, systolic and diastolic blood pressure and changes in nocturnal blood pressure dipping), PSG-derived parameters (AHI, mean nocturnal oxygen saturation, oxygen desaturation index, sleep efficiency and sleep structure), PVT reaction time, subjective wellbeing, questionnaire evaluations and overnight change in different segments of fluid volume with 2 weeks acetazolamide compared with placebo treatment.

\section{Safety outcomes}

Participants will have blood routine test, liver and renal function test and ECG at the end of each 2-week treatment periods. They will also be asked to complete an adverse event report daily, which includes information about drug-related side effects. Safety outcomes and drug-related side effects will be assessed and reported in the publication.

\section{Sample size estimation}

The minimally important difference in 24-hour mean blood pressure is assumed to be $5 \mathrm{~mm} \mathrm{Hg}$ (SD, $10 \mathrm{~mm}$ $\mathrm{Hg}$ ) based on previous studies. ${ }^{347}$ To detect this difference with a two-sided significant level of 0.05 and a power of $80 \%$, a total number of 32 participants is required. 
Therefore, our aim will be to recruit 40 participants to account for potential dropouts.

\section{Data management and monitoring}

A standardised case report form (CRF) has been specifically developed for the current study. Therefore, all studyrelevant data will be written down in the paper CRF. To avoid data loss, data will be enter in an excel database after each completed study visit and timely double-checked by another researcher. A unique study ID will be assigned for each participant and only authorised researchers will have access to the database. Given the short period of each intervention and low risk of the trial, a data monitoring committee will not be formed. However, regular data review will be performed to minimise adverse events and other unintended effects.

\section{Statistical analysis}

Data will be presented with mean (SD) and medians (quartiles) for normal and non-normal distribution. The primary outcome will be analysed according to the intention-to-treat principle including data from all randomised participants. Missing data will be replaced by multiple imputations using linear regression models with chained equations. A per protocol analysis of the primary and all secondary outcomes on available data from participants completing the study will also be performed. The effect of acetazolamide will be evaluated by computing unadjusted mean differences with $95 \%$ CIs between the effects of 2 weeks acetazolamide compared with placebo treatment using mixed linear regression analyses. Number and proportions of participants with side effects, adverse events and serious adverse events will also be reported. All statistical analyses will be two-sided and the statistical significance will be set at $\mathrm{p}<0.05$.

\section{PATIENT AND PUBLIC INVOLVEMENT}

Patients and the public will not directly be involved in the design, conduct, reporting or dissemination plans of the research. All patients will be informed that the dissemination of results will be accessible on request.

\section{ETHICS AND DISSEMINATION}

The protocol was approved by the West China Hospital of Sichuan University Biomedical Research Ethics Committee (Ethnic number: 2021(80)). The study will start to recruit participants in spring 2022. Dissemination of the results include presentations at conferences and publications in peer-reviewed journals.

\section{DISCUSSION}

OSA and systemic arterial hypertension are two highly prevalent diseases in lowlanders worldwide, and the conditions are also estimated to be highly prevalent in highland residents. Moreover, OSA is a well-known risk factor for hypertension. Acetazolamide has been proven to be an effective drug to reduce blood pressure in healthy trekkers acutely exposed to high altitude and in patients with OSA with hypertension at low altitude, however, the effect of acetazolamide on blood pressure and OSA in highlanders remains to be elucidated. We hypothesise that in highlander with OSA, acetazolamide could reduce blood pressure, improve sleep-disordered breathing and oxygen saturation, and result in improved cognitive performance. Possible underlying mechanisms related to improved blood pressure due to acetazolamide therapy might be acetazolamide-related reduction in the $\mathrm{CO}_{2}$ to $\mathrm{HCO}_{3}{ }^{-}$conversions by inhibiting the synthesis of carbonic anhydrase, resulting in metabolic acidosis and increased minute ventilation. The increased ventilation might increase the oxygen saturation and stabilise the breathing pattern by left shifting the $\mathrm{CO}_{2}$-ventilation response curve, which, therefore, may prevent periodic breathing. Another pathway might be that acetazolamide inhibits the activity of carbonic anhydrase in renal proximal convoluted tubules. This might result in a diuretic role by reducing $\mathrm{H}^{+}$production and $\mathrm{Na}^{+}$reabsorption, and increase of excretion of $\mathrm{Na}^{+}$, water and carbonate. This diuretic effect of acetazolamide could reduce the fluid transfer from the legs to neck by reducing the fluid accumulation from daytime orthostatic gravity and associated sleep-disordered breathing at night. Moreover, the reduction of body fluid has been directly correlated with blood pressure reductions in previous studies. Due to the expected large prevalence of OSA at high altitude, the results of this study may address an important gap of knowledge and may provide evidence of a simple, cheap and worldwide accessible medication to treat OSA at high altitude.

Contributors LT, MF, TL and XT conceived the concept of the manuscript and design of the work. LT and MF drafted the manuscript. All authors discussed, read and revised the manuscript, and approved its final version.

Funding This work was supported by the National Natural Science Foundation of China (U21A20335, 82100107).

Competing interests None declared.

Patient and public involvement Patients and/or the public were not involved in the design, or conduct, or reporting, or dissemination plans of this research.

Patient consent for publication Consent obtained directly from patient(s).

Provenance and peer review Not commissioned; externally peer reviewed.

Open access This is an open access article distributed in accordance with the Creative Commons Attribution Non Commercial (CC BY-NC 4.0) license, which permits others to distribute, remix, adapt, build upon this work non-commercially, and license their derivative works on different terms, provided the original work is properly cited, appropriate credit is given, any changes made indicated, and the use is non-commercial. See: http://creativecommons.org/licenses/by-nc/4.0/.

ORCID iD

Xiangdong Tang http://orcid.org/0000-0001-6876-3328

\section{REFERENCES}

1 Dempsey JA, Veasey SC, Morgan BJ, et al. Pathophysiology of sleep apnea. Physiol Rev 2010;90:47-112.

2 Young T, Palta M, Dempsey J, et al. The occurrence of sleepdisordered breathing among middle-aged adults. N Engl J Med 1993;328:1230-5. 
3 Heinzer R, Vat S, Marques-Vidal P, et al. Prevalence of sleepdisordered breathing in the general population: the HypnoLaus study. Lancet Respir Med 2015;3:310-8.

4 Deegan PC, McNicholas WT. Pathophysiology of obstructive sleep apnoea. Eur Respir J 1995;8:1161-78.

5 White LH, Bradley TD. Role of nocturnal rostral fluid shift in the pathogenesis of obstructive and central sleep apnoea. J Physiol 2013;591:1179-93.

6 Perger E, Blaise S, Vermorel C, et al. Subjects with venous insufficiency have high risk of obstructive sleep apnea in relationship to fluid shift. Sleep Med 2020;72:135-7.

7 Lyons OD, Inami T, Perger E, et al. The effect of fluid overload on sleep apnoea severity in haemodialysis patients. Eur Respir $J$ 2017;49. doi:10.1183/13993003.01789-2016. [Epub ahead of print: 0504 2017].

8 Torres G, Sánchez-de-la-Torre M, Barbé F. Relationship between OSA and hypertension. Chest 2015;148:824-32.

9 Lavie P, Herer P, Hoffstein V. Obstructive sleep apnoea syndrome as a risk factor for hypertension: population study. BMJ 2000;320:479-82.

10 Hou H, Zhao Y, Yu W, et al. Association of obstructive sleep apnea with hypertension: a systematic review and meta-analysis. J Glob Health 2018:8:010405.

11 Simancas-Racines D, Arevalo-Rodriguez I, Osorio D, et al. Interventions for treating acute high altitude illness. Cochrane Database Syst Rev 2018;6:CD009567.

12 León-Velarde F, Maggiorini M, Reeves JT, et al. Consensus statement on chronic and subacute high altitude diseases. High Alt Med Biol 2005:6:147-57.

13 Schwartz NG, Rattner A, Schwartz AR, et al. Sleep disordered breathing in four resource-limited settings in Peru: prevalence, risk factors, and association with chronic diseases. Sleep 2015;38:1451-9.

14 Pham LV, Meinzen C, Arias RS, et al. Cross-Sectional comparison of sleep-disordered breathing in native Peruvian highlanders and lowlanders. High Alt Med Biol 2017;18:11-19.

15 Boos CJ, Vincent E, Mellor A, et al. The effect of high altitude on central blood pressure and arterial stiffness. J Hum Hypertens 2017;31:715-9.

16 Parati G, Bilo G, Faini A, et al. Changes in $24 \mathrm{H}$ ambulatory blood pressure and effects of angiotensin II receptor blockade during acute and prolonged high-altitude exposure: a randomized clinical trial. Eur Heart J 2014;35:3113-22.

17 Parati G, Agostoni P, Basnyat B, et al. Clinical recommendations for high altitude exposure of individuals with pre-existing cardiovascular conditions. Eur Heart J 2018;39:1546-54.

18 Parati G, Revera M, Giuliano A, et al. Effects of acetazolamide on central blood pressure, peripheral blood pressure, and arterial distensibility at acute high altitude exposure. Eur Heart $J$ 2013;34:759-66.

19 Shen Y, Chang C, Zhang J, et al. Prevalence and risk factors associated with hypertension and prehypertension in a working population at high altitude in China: a cross-sectional study. Environ Health Prev Med 2017;22:19.

20 Aryal N, Weatherall M, Bhatta YKD, et al. Blood pressure and hypertension in adults permanently living at high altitude: a systematic review and meta-analysis. High Alt Med Biol 2016;17:185-93.

21 Mingji C, Onakpoya IJ, Perera R, et al. Relationship between altitude and the prevalence of hypertension in Tibet: a systematic review. Heart 2015;101:1054-60.

22 Bilo G, Acone L, Anza-Ramírez C, et al. Office and ambulatory arterial hypertension in highlanders: HIGHCARE-ANDES highlanders study. Hypertension 2020;76:1962-70.

23 Swenson ER. Pharmacology of acute mountain sickness: old drugs and newer thinking. J Appl Physiol 2016;120:204-15.

24 Low EV, Avery AJ, Gupta V, et al. Identifying the lowest effective dose of acetazolamide for the prophylaxis of acute mountain sickness: systematic review and meta-analysis. BMJ 2012;345:e6779.
25 Schmickl CN, Landry SA, Orr JE, et al. Acetazolamide for OSA and central sleep apnea: a comprehensive systematic review and metaanalysis. Chest 2020;158:2632-45.

26 Caravita S, Faini A, Lombardi C, et al. Sex and acetazolamide effects on chemoreflex and periodic breathing during sleep at altitude. Chest 2015;147:120-31.

27 Bucca CB, Brussino L, Battisti A, et al. Diuretics in obstructive sleep apnea with diastolic heart failure. Chest 2007;132:440-6.

28 Fiori CZ, Martinez D, Montanari CC, et al. Diuretic or sodiumrestricted diet for obstructive sleep apnea-a randomized trial. Sleep 2018;41. doi:10.1093/sleep/zsy016. [Epub ahead of print: 0104 2018].

29 Revol B, Jullian-Desayes I, Bailly S, et al. Who may benefit from diuretics in OSA?: a propensity score-match observational study. Chest 2020;158:359-64.

30 Eskandari D, Zou D, Grote L, et al. Acetazolamide reduces blood pressure and sleep-disordered breathing in patients with hypertension and obstructive sleep apnea: a randomized controlled trial. J Clin Sleep Med 2018;14:309-17.

31 Hoff E, Zou D, Schiza S, et al. Carbonic anhydrase, obstructive sleep apnea and hypertension: effects of intervention. J Sleep Res 2020;29:e12956.

32 Liu H-M, Chiang I-J, Kuo KN, et al. The effect of acetazolamide on sleep apnea at high altitude: a systematic review and meta-analysis. Ther Adv Respir Dis 2017;11:20-9.

33 Nussbaumer-Ochsner $\mathrm{Y}$, Latshang TD, Ulrich $\mathrm{S}$, et al. Patients with obstructive sleep apnea syndrome benefit from acetazolamide during an altitude sojourn: a randomized, placebo-controlled, double-blind trial. Chest 2012;141:131-8.

34 Latshang TD, Nussbaumer-Ochsner Y, Henn RM, et al. Effect of acetazolamide and autoCPAP therapy on breathing disturbances among patients with obstructive sleep apnea syndrome who travel to altitude: a randomized controlled trial. JAMA 2012;308:2390-8.

35 Netzer NC, Stoohs RA, Netzer CM, et al. Using the Berlin questionnaire to identify patients at risk for the sleep apnea syndrome. Ann Intern Med 1999;131:485-91.

36 Zhang JN, Peng B, Zhao TT, et al. Modification of the Epworth Sleepiness scale in central China. Qual Life Res 2011;20:1721-6.

37 Parrott AC, Hindmarch I. Factor analysis of a sleep evaluation questionnaire. Psychol Med 1978;8:325-9.

38 Quanjer PH, Stanojevic S, Cole TJ, et al. Multi-Ethnic reference values for spirometry for the 3-95-yr age range: the global lung function 2012 equations. Eur Respir J 2012;40:1324-43.

39 Berry RB, Budhiraja R, Gottlieb DJ, et al. Rules for scoring respiratory events in sleep: update of the 2007 AASM manual for the scoring of sleep and associated events. deliberations of the sleep apnea definitions Task force of the American Academy of sleep medicine. $J$ Clin Sleep Med 2012;8:597-619.

40 Tan L, Li T, Zhang Y, et al. Effect of one night of nocturnal oxygen supplementation on highland patients with OSA: a randomized, crossover trial. Chest 2021;160:690-700.

41 Kyle UG, Bosaeus I, De Lorenzo AD, et al. Bioelectrical impedance analysis--part I: review of principles and methods. Clin Nutr 2004;23:1226-43.

42 Shiota S, Ryan CM, Chiu K-L, et al. Alterations in upper airway crosssectional area in response to lower body positive pressure in healthy subjects. Thorax 2007;62:868-72.

43 Su Y-min, Makinia J, Pagilla KR. Estimation of autotrophic maximum specific growth rate constant--experience from the long-term operation of a laboratory-scale sequencing batch reactor system. Water Environ Res 2008;80:355-66.

44 Redolfi S, Yumino D, Ruttanaumpawan P, et al. Relationship between overnight rostral fluid shift and obstructive sleep apnea in nonobese men. Am J Respir Crit Care Med 2009;179:241-6.

45 Ding N, Lin W, Zhang X-L, et al. Overnight fluid shifts in subjects with and without obstructive sleep apnea. J Thorac Dis 2014;6:1736-41.

46 Basner M, Dinges DF. Maximizing sensitivity of the psychomotor vigilance test (PVT) to sleep loss. Sleep 2011;34:581-91.

47 Gottlieb DJ, Punjabi NM, Mehra R, et al. Cpap versus oxygen in obstructive sleep apnea. N Engl J Med 2014;370:2276-85. 